Review Article

\title{
A REVIEW ON LIQUID CRYSTALLINE NANOPARTICLES (CUBOSOMES): EMERGING NANOPARTICULATE DRUG CARRIER
}

\author{
S. NAVEENTAJ ${ }^{1}$, Y. INDIRA MUZIB ${ }^{2}$
}

Department of Pharmacy, Sri Padmavati Mahila Visvavidyalayam, [Women's University], Tirupati 517502, Andhra Pradesh [A. P.], India Email: naveentaj33@gmail.com

Received: 15 Aug 2019, Revised and Accepted: 24 Oct 2019

\begin{abstract}
Cubosomes are novel biocompatible drug delivery system and have honeycombed (cavernous) structures whose diameter size range from 10-500 $\mathrm{nm}$. They appear like dots, which are likely to be spherical structures. Each dot corresponds to the presence of a pore containing aqueous cubic phase in the lipid water system. Cubosomes posse's great significance in the field of cosmeceuticals and Pharmaceuticals due to its unique features and become an attractive choice of vehicle for in vivo drug delivery due to their low cost, safety, efficacy and versatility for controlled release application and functionalization. Cubosomes have a very simple method of preparation; biodegradability of selected lipids has the capability to encapsulate hydrophobic and hydrophilic substances. Cubosomes are considered to be versatile systems, and prepared cubosomes can be administrated by different ways such as oral, percutaneous and parenteral routes. On the whole, cubosomes offer high consequence in nano-based drug preparations for melanoma (skin cancer) treatment, targeted drug delivery systems and comprise a wide range of applications in many areas and are characterized by various parameters. Consequently, cubosomes are in progress forward of awareness in the Pharmaceutical division. This review article mainly focuses on the methods of preparation, advantages, and applications of cubosomes.
\end{abstract}

Keywords: Cubosomes, Amphiphilic, Drug payloads, Anti-cancer

(c) 2020 The Authors. Published by Innovare Academic Sciences Pvt Ltd. This is an open access article under the CC BY license (http://creativecommons.org/licenses/by/4.0/) DOI: http://dx.doi.org/10.22159/ijcpr.2020v12i1.36820. Journal homepage: https://innovareacademics.in/journals/index.php/ijcpr

\section{INTRODUCTION}

Cubosomes are defined as discrete, sub-micron, nano-structured particles of bicontinuous cubic liquid crystalline phase. Cubosomes possess larger surface area and have same micro structure as that parent cubic phase and the prepared cubosomes dispersions have much lower viscosity compared with bulk cubic phase. The term cubosomes first proposed by Larsson, that reflects the cubic molecular crystallography and similarity to liposomes [1] Selected lipids, surfactants and polymer molecules have both polar and non polar components, termed as amphiphilic [2].

Due to its amphiphilic nature molecules progress by the hydrophobic effect into polar solvent to spontaneously recognize and unite into a liquid crystal of nanometer scale. Thus, Cubosomes are bicontinuous cubic liquid phase surrounded by two different regions of water separated by surfactant controlled bilayers. Further these are parallel to liquid crystalline substance with cubic crystallographic symmetry and are visually isotropic, viscous and solid too. The cubic phase can split and form thermodynamically stable particulate dispersions [3].
Cubosomes set to have a great significance in nanodrug formulations. Reported studies shown that cubosomes formulation have been revealed to be safe in targeted drug delivery mainly in brain targeting [4] and the prepared cubosomal gels of fluconazole which resulted in enhanced pay drug load, entrapment efficiency, enhanced drug permeability compared with conventional gels [5]. One of the reason to select cubosomes as carrier system is that it posses high physicochemical stability of surfactant and emulsifiers than that of phospholipids which are used in the preparation of liposomes. Cubosomes are useful over other drug delivery system due to their bioavailability improvement of poorly soluble drugs and enhances skin permeation, by this it reduces the cost of therapy [6, 7]. Surfactant used in cubosomes does not require any special handling, and storage condition.

\section{Structure of cubosomes}

Cubosomes consist of honeycombed (cavernous) structures separating two internal aqueous channels and a large interfacial area. Self assembled cubosomes as active drug delivery systems are receiving much more attention and interest after the first discovery $[8,9]$.

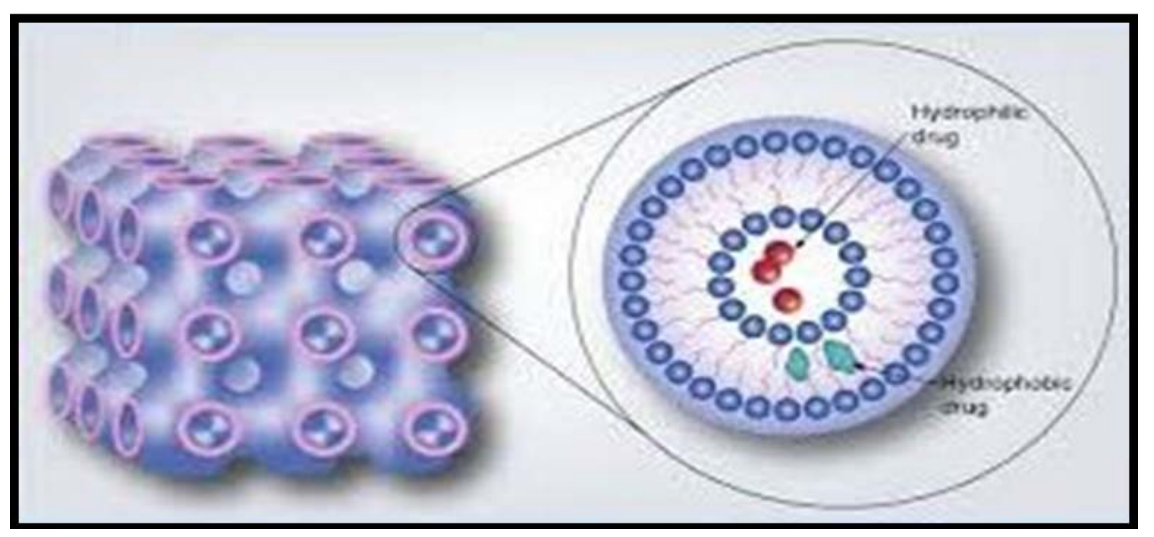

Fig. 1: Structure of cubosomes 
Cubosomes are nanoparticles whose size ranges from 10-500 nm in diameter and appear like dots, which are likely to be spherical. Each dot corresponds to the presence of pore containing aqueous cubic phase in lipid water system, which was first recognized by Luzzati and Husson using X-ray scattering technique. According to Fontell and Drew ternary systems of amphiphiles, oil and water, some monoglycerides will exhibits cubic phases. Monoglycerides are polar lipids and have poor water solubility that exhibits aqueous phase behavior, which are structurally mimicking to nonionic surfactants $[10,11]$.

Cubosomes are amphiphilic carrier system which has a capability to encapsulate both hydrophilic and lipophilic drugs. The hydrophilic drug is encapsulated inside the vesicles whereas the lipophilic drug is partitioned between the hydrophilic domains. Liposomes are produced by the self-assembly of phospholipids in aqueous phase to form bilayer which may be spherical unilamellar or multilamellar vesicles [12]. Some other phospholipid carriers like liposome may undergo some problems like degradation by hydrolysis in aqueous solution, sedimentation and aggregation on storage and cannot sterilize for clinical use. Still, chemical instability like oxidation of phospholipids was not avoided [13]. These pave the way to the discovery of non-ionic surfactant vesicles known as cubosomes [14].

Lipids have been widely used as main constituents in various drug delivery systems [15], like in formulations of liposomes, solid lipid nanoparticles, nanostructured lipid carriers, and lipid-based lyotropic liquid crystals [16]. Among them, lipid-based lyotropic liquid crystals have highly ordered, thermodynamically stable internal nanostructure, thereby offering the potential as a sustained drug release matrix. The intricate nanostructures of the cubic phase have been shown to provide diffusion controlled release of active pharmaceutical ingredients with a wide range of molecular weights and polarities. In addition, the biodegradable and biocompatible nature of lipids demonstrates the minimum toxicity and thus they are used for various routes of administration

The inimitable microstructure of cubosomes confers some desirable characteristics as far as controlled release is concerned. Firstly, the drugs of varying polarities and sizes can be entrapped in the cubic phases [17]. Because of the dual polar/nonpolar nature of the cubic phase, hydrophilic drugs are trapped in the aqueous channels while their hydrophobic counterparts will partition into the lipid bilayer compartment which will be the rate limiting step $[18,19]$. Secondly, the unique microstructure of the cubic phase controls the degree of drug release. Thus, the drug must diffuse from the three dimensional network in which both tortuosity and pore size of the aqueous nanochannels contribute to delayed/controlled release. Thirdly, the cubic mesophases are stable in vitro and may be subjected to lipolysis which facilitates ultimate dissolution in vivo. Finally, the cubic phase exhibits a bioadhesive property [20], which makes it useful for delivery of gastrointestinal, lung, nasal, oral, buccal, rectal and vaginal drugs [21].

\section{Manufacturing techniques of cubosomes}

Dispersion of the nanoparticles produced in the cubosome formation can be done by several techniques: sonication, high pressure homogenization, spontaneous emulsification, and spray drying. Sonication and high-pressure homogenization suggest the formation of complex dispersions containing vesicles and cubosomes with time dependent ratios of each particle type [22].

Cubosomes can be manufactured by two distinct methods [23]:

\section{Top down Technique}

2. Bottom up Technique

In Bottom up technique, cubosome dispersion is formed by dilution of an isotropic solution, [24] whereas in top down technique powder cubosome precursors can be achieved [25]. Liquid crystalline dispersions are prepared by selected techniques like; 1 . Cubosomes from pseudo-binary systems, 2 . Cubosomes by nucleation 3. Cubic liquid crystalline phase in the presence of hydrotropes, 4. Dry powder precursor [26].

\section{Top down technique}

It is the most frequently used procedure initially reported in 1996 by Ljusberg-Wahren [27]. Bulk cubic phase is first produced and by application of high energy such as high pressure homogenization it is processed into cubosomes nanoparticles. Bulk cubic phase resembles a clear rigid gel formed by water-swollen cross-linked polymer chains. The cubic phases differ in that they are a single thermodynamic phase and have periodic liquid crystalline structure [28]. Cubic Phases ruptures in a direction parallel to the shear direction, the energy required is proportional to the number of tubular network branches that rupture.

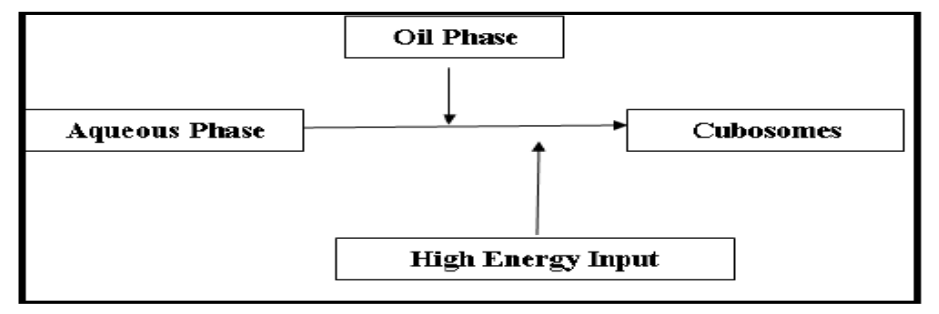

Fig. 2: Top-down technique

\section{Bottom up technique}

In this cubosomes are allowed to form or crystallize from precursors. The bottom-up approach first forms the nanostructure building blocks and then assembles them into the final material. It is more recently developed technique of cubosome development, allowing cubosomes to form and crystallize from precursors on the molecular scale length. The input factor of this technique is hydrotrope that can dissolve water insoluble lipids into liquid precursors. This is a dilution based approach that produces cubosomes with less energy input when compared to top down approach [29].

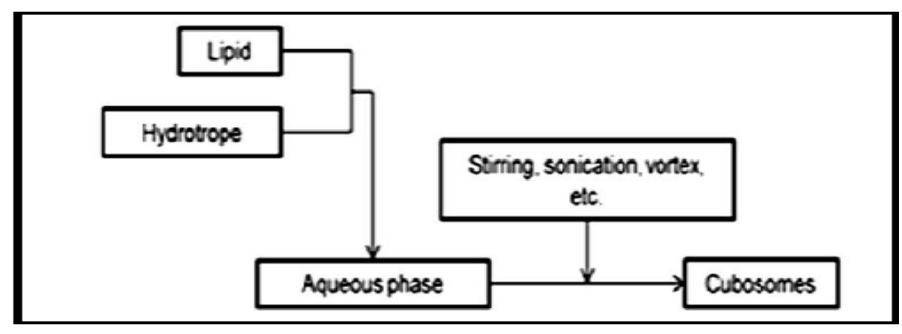

Fig. 3: Bottom up technique 


\section{Advantages of cubosomes [30-33]}

Sustained and targeted release profiles for drugs can be achieved. It has an ability to entrap both lipophilic and hydrophilic drugs and consider being thermodynamically stable for longer time, economical, cost effective procedures. Due to high internal surface area and cubic crystalline structures, it has high drug loading capacity, it involves simple preparation methods and lipids used in cubosome preparation are biocompatible and non-toxic in nature it has good bio adhesive properties and easily incorporated into product formulations. It acts as excellent solubilizers and skin permeation enhancers.

\section{Disadvantages of cubosomes [34]}

Few disadvantages with cubosomes are its high viscosity nature; large scale production is sometimes difficult. Low entrapment of water soluble drugs due to presence of high amounts of water inside cubosomes and when compared with polymer based drug delivery cubosomes do not offer controlled drug delivery on their own.

Advantages of cubosomes (Surfactant and phospholipid based carrier system) in comparison with other delivery systems [35-37]

When compared with other carrier systems cubosome drug delivery system shows enhanced drug permeation through skin for percutaneous and transdermal delivery. This platform is considered to helpful in delivery of large and diverse groups like proteins, peptide molecules. The toxicological profiles of the phospholipids are well documented in the scientific Literature noticed low risk profiles. High market attractiveness for products with proprietary technology. Relatively simple to manufacture with no complicated technical investments required for Production of cubosomes. This vesicular system is passive and noninvasive; it is available for immediate commercialization.

\section{Applications}

\section{Topical drug delivery systems [38]}

Cubic phases have bioadhesive property, so that they can conveniently use in topical and mucosal depositions and delivery of diverse drugs. Topical delivery systems are based on the exploitation of unique properties of liquid crystal (LC) and liquid crystal nanoparticle (LCNP) technologies. Topical drug delivery systems are unique in situ forming bioadhesive LC systems facilitate controlled and effective drug delivery to mucosal surfaces (buccal, ophthalmic, vaginal and others). This attractive system forms a thin surface film at mucosal surfaces consisting of a liquid crystal matrix nanostructure achieving an optimal delivery profile and provides excellent temporary protection for sore and sensitive skin.

\section{Oral drug delivery system [39]}

Cubosomes deal with the varied challenges in oral delivery of numerous promising compounds including poor absorption, poor aqueous solubility, and huge molecular size. In an alternative application large proteins have been encapsulated for local activity in the gastrointestinal region. Liquid crystalline nanoparticles based technology carriers can be combined with controlled release and targeting functionalities. The particles are deliberate to form in situ in a controlled rate, which facilitates an effective in vivo distribution of the drug. Cubosomes technology carriers can also be released at different absorption sites, for example in the region of upper or lower intestine, which is essential for the drugs that have narrow regional absorption window.

\section{As sustained release behavior [40]}

Latest patent activity on cubosome use in personal care product areas like skin care, hair care, cosmetics, and antiperspirants. Regardless of recent activity, there remains a lack of the practical elements like manufacturing scalability and material customization that is necessary to lead formulators to consider using cubosomes in commercial products. The cubic phase has been shown to provide a vehicle for several in vivo delivery routes, including depot, transdermal, mucoadhesion and ophthalmic. An extensive variety of drugs with different physicochemical properties have been incorporated in cubosomes, and their sustained release behavior was also carried out. Sustained behavior of cubosomes was because of cubosome remnant particles. Monoglyceride based cubosome dispersion can be proposed for topical use, such as for perctuneous or mucosal applications.

\section{Melanoma (cancer) therapy [41]}

In recent times few anticancer drugs have been effectively encapsulated in cubosomes and evaluated Physicochemically. The distinctive structure of this promising nanocarrier suggests its application in melanoma therapy. In order to specifically target nanomedicines to tumors, different ways have been envisaged, with passive and active targeting of cancer cells having been shown to be valid approaches in preclinical and clinical studies. Passive targeting exploits the Pathophysiological properties of the tumour vasculature which is generally highly disorganised with enlarged gap junctions between endothelial cells and compromised lymphatic drainage allowing for the extravasation of nanocarriers with sizes up to several hundred nanometer ranges. Objects of this size cannot pass through the tight junctions that exist within the endothelial cell lining of the vessels of healthy tissues.

Table 1: Comparison between drug alone and drug encapsulated cubosomes

\begin{tabular}{ll}
\hline Drug alone & Drug encapsulated in cubosomes \\
\hline Fail to distinguish normal cells from cancer cells & Distinguish normal cells from cancer cells \\
Less biodistrubution & More biodistrubution \\
Low efficacy & More efficacy \\
Severe toxic side effects & Less side-effects. \\
\hline
\end{tabular}

Table 2: Different drugs encapsulated in cubosomes to achieve sustain and controlled release [44-50]

\begin{tabular}{|c|c|c|c|c|}
\hline S. No. & Researcher & Drug & Category & Associated disease \\
\hline 1 & Omar et al. & Clotrimazole & Antifungal & Vagina, mouth, and skin infection \\
\hline 2 & Damani et al. & Clindamycin phosphate & Antibiotics & Peritonitis, staphylococcal bone and joint infection \\
\hline 3 & Engstrom et al. & Clomethiazole & Psychotropic & Insomnia \\
\hline 4 & Nielsen et al. & $\begin{array}{l}\text { Indomethacin Isosorbide } \\
\text { mononitrate Lidocaine- } \\
\text { hydrochloride }\end{array}$ & $\begin{array}{l}\text { NSAIDs Anti-anginal Aural } \\
\text { Preparation }\end{array}$ & $\begin{array}{l}\text { Gout, rheumatoid arthritis Angina pectoris Fungal } \\
\text { infection of external ear }\end{array}$ \\
\hline 5 & Boyd et al. & $\begin{array}{l}\text { Diazepam Rifampicin } \\
\text { Griseofulvin Propofol }\end{array}$ & $\begin{array}{l}\text { Sedative-hypnotic Bactericidal } \\
\text { antibiotic Antifungal Hypnotic }\end{array}$ & $\begin{array}{l}\text { Anxiety, insomnia, seizures Tuberculosis Fungal } \\
\text { infection of skin Procedural sedation. }\end{array}$ \\
\hline 6 & Engstrom et al. & Gramicidin Insulin & $\begin{array}{l}\text { Topical steroidHypo/Hyper } \\
\text { glycemics }\end{array}$ & Corticosteroid sensitive dermatoses Diabetes mellitus \\
\hline 7 & Sadhale et al. & $\begin{array}{l}\text { Cefazolin Cefuroxime } \\
\text { Prilocaine }\end{array}$ & $\begin{array}{l}\text { Antibiotics Antibiotics Local } \\
\text { anesthetic }\end{array}$ & $\begin{array}{l}\text { Genito-urinary, respiratory tract infection. Meningitis } \\
\text { bone and soft tissue infection. In dentistry }\end{array}$ \\
\hline
\end{tabular}




\section{As a drug delivery vehicle [42]}

The rapid growth of the life-sciences industry is likely to drive previously "exotic" delivery vehicles and ingredients into broader market places, such as personal care and consumer products. Consequently, self-assembled surfactant phases have been broadly examined for compatibility with abundant medical active ingredients and their applications. The number of research in connection with cosmetic companies like L'Oreal and Nivea are trying to utilize cubosome particles as oil-in-water emulsion stabilizers and pollutant absorbents in cosmetics.

\section{Intravenous drug delivery systems [43]}

Lipid nanoparticles comprising internal liquid crystal structures of curved lipid membranes are used to solubilize encapsulate and transport medications to disease areas within the body. While emulsions and liposomes have found use as intravenous carriers in drug products, liquid crystal nanoparticle structures increases payloads of peptides, proteins and various insoluble small molecules, and are perfect carriers for injection or infusion of many actives.

\section{CONCLUSION}

Cubosomes are novel surfactant based carrier system which involves simple preparation methods and has a composition of simple mixture of biologically compatible lipids and water which are well suited for the body tissues. Cubosomes offers increased flexibility for product development and this act as excellent solubilizers, compared with conventional lipid or non-lipid carriers. When compared with conventional carrier systems overall, cubosomes have great potential in drug nanoformulations for melanoma therapy owing to their potential advantages, including high drug payloads, high internal surface area and cubic crystalline structures, relatively simple preparation method, biodegradability of lipids, the ability of encapsulating hydrophobic, hydrophilic substances, targeting and controlled release of bioactive agents like proteins and drugs. Moreover, in many studies it is reported that drug incorporated cubosomes have utility as a controlled and sustained release drug carrier.

\section{AUTHORS CONTRIBUTIONS}

All the authors have contributed equally.

\section{CONFLICT OF INTERESTS}

Declare none

\section{REFERENCES}

1. Andersson S, Jacob M, Ladin S, Larsson K. Structure of the cubosomes-a closed lipid bilayer aggregate. Zeitschrift Fur Kristallographie 1995;210:315-8.

2. Schwarz US, G Gompper. Bicontinous surfaces in self assembling amphiphilic systems. Lect Notes Phys 2002;600: 107-51.

3. Saurabh Bansal, Chandan Prasad Kashyap, Geeta Aggarwal. A comparative review on vesicular drug delivery system and stability issues. Int J Res Pharma Chem 2012;2:704-13.

4. Yosra SRE, Samar ME, Doaa AA, Ossama YA. Novel piperineloaded tween-integrated monoolein cubosomes as braintargeted oral nanomedicine in Alzheimer's disease: pharmaceutical,biological and toxicological studies. Int J Nanomed 2015;10:5459-73.

5. Ruchi S, Gurvinder K, Deepak NK. Fluconazole loaded cubosomal vesicles for topical delivery. Int J Drug Dev Res 2015; 7:32-41.

6. Sindhumol PG, Thomas M, Mohancharan PS. Phytosomes: a novel dosage form for enhancement of bioavailability of botanicals and nutraceuticals. Int J Pharm Pharm Sci 2010;4:10-4.

7. Schreier H, J Bouwstra. Liposomes and niosomes as topical drug carriers: dermal and transdermal delivery. J Controlled Release 1994;30:1-15.

8. Drummond CJ, Fong C. Surfactant self-assembly objects as novel drug delivery vehicles. Curr Opin Colloid Interface Sci 2000;4:449-56.s
9. Karami Z, Hamidi M. Cubosomes: remarkable drug delivery potential. Drug Discovery Today 2016;21:789-801.

10. Luzzati V, Tardieu A, Gulik Krzywicki T, Rivas E, Reiss Husson F. Structure of cubic phases of lipid-water systems. Nature 1968;220:485-8.

11. Fontell K, Mandell L, Ekwall P. Some isotropic mesophases in the systems containing amphiphilic compounds. Acta Chem Scanda 1968;22:3209-23.

12. Bouwstra JA, Honeywell-Nguyen PL. Vesicles as a tool for transdermal and drug delivery system Drug Discovery Today Drug Delivery Nanotech 2005;2:67-74.

13. Gustafsson J, Ljusberg Wahren H, Almgren M, Larsson K. Submicron particles of reversed lipid phases in water stabilized by a nonionic amphiphilic polymer. Langmuir 1997;13:6964-71.

14. Uchegbu FI, Vyas PS. Nonionic surfactant based vesicles in drug delivery. Int J Pharm 1998;7:33.

15. Jens Birk lauridsen. Food emulsifiers: surface activity, edibility manufacture, composition and application. J Am Oil Chem Soc 1976;53:400-7.

16. Larsson K. Cubic liquid-water phases: structures and biomembrane aspects. J Phys Chem 1989;93:7301-14.

17. Bhosale RR, Osmani RA, Harkare BR, Ghodake PP. Cubosomes. the inimitable nanoparticle drug carriers. Sch Acad J Pharm 2013;2:481-6.

18. Hyde ST, Andersson S, Ericsson B, Larsson K. A cubic structure consisting of a lipid bilayer forming an infinite periodic minimal surface of the gyroid type in the glycerol monooleate water system. Z Kristallogr 1984;168:213-9.

19. Clogston J, Craciun G, Hart DJ, Caffrey M. Controlling release from the lipidic cubic phase by selective alkylation. J Controlled Release 2005;102:441-61.

20. Nielsen L, Schubert L, Hansen J. Bioadhesive drug delivery systems. Characterisation of mucoadhesive properties of systems based on glyceryl mono-oleate and glyceryl monolinoleate. Eur J Pharm Sci 1998;6:231-9.

21. Wu H, Li J, Zhang Q, Yan X, Guo L, Gao X, et al. A novel small odorranalectin-bearing cubosomes: preparation, brain delivery and pharmacodynamic study on amyloid-b25-35treated rats following intranasal administration. Eur J Pharma Bio Pharm 2012;80:368-78.

22. Almgren $\mathrm{M}$, Edwards $\mathrm{K}$, Gustafsson J. Cryotransmission electron microscopy of thin vitrified samples. Curr Opin Colloid Interface Sci 1996;1:270-8.

23. Lynch ML, Spicer PT. Cubic liquid crystalline composition and methods of preparation. USA Patent App; 2002.

24. Radiman SC Toprakcioglu, $\mathrm{T}$ Mcleish. Rheological study of ternary cubic phases. Langmuir 1994;10:61-7.

25. Spicer PT, Small WB, Lynch ML, Burns JL. Dry powder precursors of cubic liquid crystalline nanoparticles (cubosomes). J Nanopart Res 2002;4:297-311.

26. Patrick TS, Spicer L, William BS, Matthew LL, Janet LB. Dry powder precursors of Cubic liquid crystalline nanoparticles (cubosomes). J Nanopart Res 2002;4:297-311.

27. Gustafsson J, Ljusberg Wahren H, Almgren M, Larsson K. Cubic lipid water phase dispersed into submicron particles. Langmuir 1996;12:4611-13.

28. Spicer PT, Small WB, Lynch ML, Burns JL. Dry powder precursors of Soft cubic liquid crystalline nanoparticles (cubosomes). J Nanoparticles Res 2002;4:297-311.

29. MahaKa Khalifa. Miconazole nitrate based cubsosome hydrogels for topical application. Int J Drug Delivery 2015;7:1-12.

30. Cordero JA, Alarcon L, Escribano E, Obach R, Domenech J. A comparative study of the transdermal penetration of a series of non-steroidal anti-inflammatory drugs. J Pharm Sci 1997;86:503-8.

31. Muller RH, Petersen RD, Hommoss A, Pardeike J. Nanostructred lipid carriers (NLC) in cosmetic dermal products. Adv Drug Delivery Rev 2007;59:522-30.

32. Komal Tilekar, Prashant Khade, Sujit Kakade, Sachin Kotwal, Ravindra Patil. Cubosomes-a drug delivery system. IJPCBS 2014;4:812-24.

33. Geraghty PB, Attwood D, Collett JH, Sharma H, Dandiker Y. An investigation of the parameters influencing the bioadhesive properties of Myverol 18-99/water gels. Biomaterials 1997;18:63-7. 
34. Murthy SK. Nanoparticles in modern medicine: state of the art and future challenges. Int J Nanomed 2007;2:129-41.

35. YR Hundekar, JK Saboji, SM Patil, BK Nanjwade. Preparation and evaluation of diclofenac sodium cubosomes for percutaneous administration. World J Pharm Pharm Sci 2014;3:523-39.

36. Gandhi A, Maji P. Self-emulsifying drug delivery system: an approach for enhancement of bioavailability of poorly water soluble drugs. Int J Pharm Integrated Life Sci 2013;1:1-16.

37. Anbarasan BA, Fatima Grace XA, Shanmuganathan SA. An overview of cubosomes. Smart drug delivery system. Sri Ramachandra J Med 2015;8:1-4.

38. Ljusberg Wahren H, Engstrom S, Gustafsson A. Bioadhesive properties of the monoolein-water system, Pharm Tech Eur 1995;11:14-7.

39. Yosra SRE, Samar ME, Doaa AA, Ossama YA. Novel piperineloaded tween-integrated monoolein cubosomes as braintargeted oral nanomedicine in Alzheimer's disease: pharmaceutical, biological and toxicological studies. Int J Nanomed 2015;10:5459-73.

40. Deepak P, Dharmesh S. Cubosomes: a sustained drug delivery carrier. Asian J Res Pharm Sci 2011;1:59-62.

41. Bei D, Meng J, Youan BC. Engineering nanomedicines for improved melanoma therapy: progress and promises. Nanomedicine (Lond) 2010;5:1385-99.
42. Afriat I, Biatry B. Use of cubic gel particles as agents against pollutants, especially in a cosmetic composition. Eur Pat Appl (L'Oreal, Fr.). Ep; 2001.

43. Rizwan SB. Bicontinuous cubic liquid crystals as sustained delivery systems for peptides and proteins. Expert Opin Drug Delivery 2010;7:1133-44.

44. Omar SM, Ismail A, Hassanin KD, Dawoud SH. Formulation and evaluation of cubosomes as skin retentive system for topical delivery of clotrimazole. J Adv Pharm Res 2019;3:68-82.

45. Damani NC. Sustained release applications for treating periodontal disease. Eur Patent EP 0429224; 1991.

46. Engstrom S, Ljusberg Wahren H, Gustafsson A. Bioadhesive properties of the monoolein-water system. Pharm Tech Eur 1995;11:14-7.

47. Engstrom S, Lindman B, Larsson K. Method of preparing controlled release preparations for biologically active materials and resulting composition, US patent, 5151272; 1992.

48. Boyd BJ. Characterisation of drug release from cubosomes using the pressure ultrfiltration method. Int J Pharm 2003;260:239-47.

49. Engstorm S. Drug delivery from cubic and other lipid-water phases. Lipid Techonol 1990;2:42-5.

50. Sadhale Y, Shan JC. Glyceryl monooleate cubic phase gel as chemical stability enhancer of cefazolin and cefuroxime. Pharm Dev Technol 1998;3:549-56. 